\section{Metastasis-related miRNAs: a new way to differentiate patients with higher risk?}

\author{
"If the detection of miRNAs overcomes the limitations of today's \\ biomarkers, we will be able, in the near future, to improve diagnosis, \\ treatment, prognosis in cancer patients."
}

\section{Athina Markou', Evi Lianidou' \& Vassilis Georgoulias*,2}

\begin{abstract}
Metastasis refers to the spread of cancer cells to distant organs in the body. Although metastasis is the major cause of mortality in cancer patients, there are still many unanswered questions at the cellular and molecular level. miRNAs seem to join the pieces of this huge puzzle and may offer the unique potential to understand in a better way the biology of metastasis and resistance mechanisms to established therapies. This editorial focuses on the last advances on the role of microRNAs in the regulation of metastasis as well as in cancer monitoring and therapy.
\end{abstract}

miRNAs are small molecules (22-25 nt) that play an important role in gene regulation, as they can target multiple 'genes' for the 'fine tuning' of their expression. The first association between miRNAs and cancer was shown in 2002 by George Calin and Carlo Croce, who, after 7 years of research, revealed that two miRNAs were causally involved in the pathogenesis of chronic lymphocytic leukemia [1]. Today, 12 years after this important finding, a plethora of more than 1800 human miRNAs have been unveiled, and their role as key players in several stages of metastasis has been demonstrated in several studies [2].

miRNAs could be paralleled as a coin with two sides, as they seem to control metastasis in various cancers either as metastasis-suppressive miRNAs or as metastasis-promoting miRNAs. It is also important to note that the same miRNA can act in a metastasis-suppressive or metastasis-promoting role, depending on the target. Their role in tumor invasion and metastasis was first mentioned by Bob Weinberg's group, who documented a novel function for miR-10b in promoting invasion and metastasis by regulating the expression of the prometastatic small GTPase RhoC [3]. Since then, several groups started to investigate the role of miRNAs in each step of metastasis and there has been an explosion in the number of publications concerning metastasis-related miRNAs [4].

Today the list of miRNAs involved in metastasis has increased exponentially; some of them are established as antimetastatic miRNAs, such as miR-31, miR-335, miR-451, miR-206, miR-126 and other prometastatic miRNAs such as miR-10b, miR-373, miR-520c, miR-21, miR-143 and miR-182.

Due to their small size and their remarkable stability in several types of clinical samples such as serum, plasma, formalin-fixed, paraffin-embedded and fresh-frozen tissues, miRNAs can serve

\section{KEYWORDS}

- circulating miRNAs • liquid biopsy

- metastasis • miRNAs • tumor biomarkers
'Analysis of Circulating Tumor Cells, Lab of Analytical Chemistry, Department of Chemistry, University of Athens, Athens, Greece

2Laboratory of Tumor Cell Biology, Medical School, University of Crete, Heraklion, Greece

*Author for correspondence: Tel.: +30 2810 392783; Fax: +30 2810 543601; georgsec@med.uoc.gr 
"It is clear that despite their small size, miRNAs will play a significant role in the fight against cancer in the future." as novel tumor biomarkers [5,6]. It has also been shown that miRNA expression profiles are similar between paired fresh-frozen tissues and formalin-fixed, paraffin-embedded samples. Nowadays, many groups have focused on studying the expression profile of miRNAs in different types of cancer. The main questions arising are:

- Could we distinguish high-risk from low-risk patients by using only the expression pattern of specific metastasis-related miRNAs?

- Could miRNAs expression profile be used in the clinical setting?

- Do miRNAs have a potential as diagnostic, prognostic and predictive biomarkers?

- Could miRNAs be used in cancer therapeutics?

These questions are difficult to answer, but scientific evidence is continuously supporting the use of miRNAs toward these directions. It is now clear that miRNAs are suitable for discriminating malignant from nonmalignant tissues in breast, colon, lung, renal, prostate and other types of cancer [7]. Moreover, by using miRNA expression profiles, poorly differentiated tumors could be classified with better accuracy than by using mRNA expression profiles [8], and this finding is a very strong indication that miRNA profiling could be used in cancer diagnosis. Large high-throughput studies have recently revealed that miRNA profiling has not only the potential to clarify tumors, but can additionally predict patient outcome with high accuracy [9]. The potential of miRNAs in predicting patient outcome and prognosis is evident in several studies [10]. It has been clear that overexpression of miR-21 correlates with the presence of metastasis in non-small-cell lung cancer patients, while underexpression of let-7 and overexpression of miR-155 correlates with poor postoperative survival in lung cancer patients [11,12].

Increasing evidence indicates that the introduction of specific miRNAs into cancer cells and tissues induces favorable therapeutic responses. Several preclinical animal studies provide evidence demonstrating that the administration of miRNAs induces therapeutic response by blocking or reducing tumor growth [13]. Not surprisingly, miRNA-targeting therapies are an area of intense interest to pharmaceutical companies and many such compounds are in preclinical and clinical development for a variety of indications. Although, for the most part, miRNA therapeutics is still in a preclinical development phase. Two therapies have reached clinical trials: one is LNA anti-miR [14] and the other is miRNA mimic therapy, which seems to be the new insight for cancer therapy. Recently, MRX34 entered Phase I clinical trials in patients with advanced hepatocellular carcinoma [15].

Tumor resistance remains a major unresolved obstacle to successful chemotherapy. miRNAs play an important role in the development of chemosensitivity or chemoresistance mechanisms in different types of cancer, as miRNAs can change the cellular response to a specific drug or class of drugs, not only through survival or apoptotic signaling but also by interfering with specific drug targets [16,17]. A strong association between low miR-26 expression and both prognosis and response to IFN therapy in patients with hepatocellular carcinoma has been reported [18]. Nevertheless, the first clinical trial evaluating miRNA levels during exposure to targeted therapies is ongoing [19]. These results will show if changes in miRNA expression levels can have a predictive function and can provide the demonstration of their causative role in the onset of resistance or even identify novel therapeutic targets [17].

Mitchell et al. were the first who clearly showed that circulating miRNAs originate from cancer tissues and are protected from endogenous RNase activity, reflecting the potential of circulating miRNAs to serve as circulating tumor biomarkers [20]. The presence of circulating miRNAs in body fluids may represent a gold mine of noninvasive tumor biomarkers, since deregulated miRNA expression is an early event in tumorigenesis. Measuring circulating miRNA levels may also be useful for early cancer detection, which can contribute greatly to the success of treatment. More than 200 studies so far have assessed the potential use of serum or plasma miRNAs as biomarkers in different types of cancer. Interestingly, highly expressed circulating miRNAs from cancer patients have been reported to return to a normal level after tumor resection [21,22].

The finding that miRNAs are included in exosomes could explain their stability in blood [23]. Very recently Melo et al. have shown that the presence of specific miRNAs in exosomes could unravel the possible role of cancer exosomes in 
inducing an oncogenic 'field effect' that further subjugates adjacent normal cells to participate in cancer development and progression [24].

It is clear that despite their small size, miRNAs will play a significant role in the fight against cancer in the future. If the detection of miRNAs overcomes the limitations of today's biomarkers, we will be able, in the near future, to improve diagnosis, treatment, prognosis in cancer patients.

\section{Financial \& competing interests disclosure}

The authors have no relevant affliations or financial involvement with any organization or entity with a financial interest in or financial conflict with the subject matter or materials discussed in the manuscript. This includes employment, consultancies, honoraria, stock ownership or options, expert testimony, grants or patents received or pending or royalties.

No writing assistance was utilized in the production of this manuscript.

\section{References}

1 Calin GA, Dumitru CD, Shimizu M et al. Frequent deletions and down-regulation of micro- RNA genes miR15 and miR16 at 13q14 in chronic lymphocytic leukemia. Proc. Natl Acad. Sci. USA 99(24), 15524-15529 (2002).

2 Pencheva N, Tavazoie SF. Control of metastatic progression by microRNA regulatory networks. Nat. Cell Biol. 15(6), 546-554 (2013).

3 Ma L, Teruya-Feldstein J, Weinberg RA. Tumour invasion and metastasis initiated by microRNA-10b in breast cancer. Nature 449 (7163), 682-688 (2007).

4 White NM, Fatoohi E, Metias M et al. Metastamirs: a stepping stone towards improved cancer management. Nat. Rev. Clin. Oncol. 8(2), 75-84 (2011).

5 Cortez MA, Bueso-Ramos C, Ferdin J et al. MicroRNAs in body fluids - the mix of hormones and biomarkers. Nat. Rev. Clin. Oncol. 8(8), 467-477 (2011).

6 Schwarzenbach H, Nishida N, Calin GA et al. Clinical relevance of circulating cell-free microRNAs in cancer. Nat. Rev. Clin. Oncol. 11(3), 145-156 (2014).

7 Wang H. Predicting cancer-related miRNAs using expression profiles in tumor tissue. Curr. Pharm. Biotechnol. 15(5), 438-444 (2014).
8 Lu J, Getz G, Miska EA et al. MicroRNA expression profiles classify human cancers. Nature 435 (7043), 834-838 (2005).

9 Chen X, Liu MX, Yan GY. RWRMDA: predicting novel human microRNA-disease associations. Mol. Biosyst. 8(10), 2792-2798 (2012).

10 Mishra PJ. Non-coding RNAs as clinical biomarkers for cancer diagnosis and prognosis. Expert Rev. Mol. Diagn. 14(8), 917-919 (2014).

11 Takamizawa J, Konishi H, Yanagisawa K et al. Reduced expression of the let-7 microRNAs in human lung cancers in association with shortened postoperative survival. Cancer Res. 64(11), 3753-3756 (2004).

12 Yanaihara N, Caplen N, Bowman E et al. Unique microRNA molecular profiles in lung cancer diagnosis and prognosis. Cancer Cell 9(3), 189-198 (2006).

13 Ma L, Reinhardt F, Pan E et al. Therapeutic silencing of miR-10b inhibits metastasis in a mouse mammary tumor model. Nat. Biotechnol. 28(4), 341-347 (2010).

14 Sanchez-Nino MD, Ortiz A. HCV infection and miravirsen. N. Engl. J. Med. 369(9), 877-878 (2013).

15 A Multicenter Phase I Study of MRX34, MicroRNA miR-RX34 Liposomal Injection. https://clinicaltrials.gov
16 Ma J, Dong C, Ji C. MicroRNA and drug resistance. Cancer Gene Ther. 17(8), 523-531 (2010).

17 Migliore C, Giordano S. Resistance to targeted therapies: a role for microRNAs? Trends Mol. Med. 19(10), 633-642 (2013).

18 Ji J, Shi J, Budhu A et al. MicroRNA expression, survival, and response to interferon in liver cancer. $N$. Engl. J. Med. 361(15), 1437-1447 (2009).

19 ClinicalTrials.gov. www.clinicaltrials.gov

20 Mitchell PS, Parkin RK, Kroh EM et al. Circulating microRNAs as stable blood-based markers for cancer detection. Proc. Natl Acad. Sci. USA 105(30), 10513-10518 (2008).

21 Heneghan HM, Miller N, Lowery AJ et al. Circulating microRNAs as novel minimally invasive biomarkers for breast cancer. Ann. Surg. 251(3), 499-505 (2010).

22 Yamamoto Y, Kosaka N, Tanaka M et al. MicroRNA-500 as a potential diagnostic marker for hepatocellular carcinoma. Biomarkers 14(7), 529-538 (2009).

23 Bail S, Swerdel M, Liu H et al. Differential regulation of microRNA stability. RNA 16(5), 1032-1039 (2010).

24 Melo SA, Sugimoto H, O'Connell JT et al. Cancer exosomes perform cell-independent microRNA biogenesis and promote tumorigenesis. Cancer Cell 26(5), 707-721 (2014). 